\title{
PT-Symmetric planar devices for field transformation and imaging
}

\author{
C A Valagiannopoulos ${ }^{1,3}$, F Monticone $^{2,3}$ and A Alù ${ }^{2}$ \\ ${ }^{1}$ Department of Physics, School of Science and Technology, Nazarbayev University, 53 Qabanbay Batyr \\ Ave, Astana, KZ-010000, Kazakhstan \\ ${ }^{2}$ Department of Electrical and Computer Engineering, The University of Texas at Austin, 1616 Guadalupe \\ St., Austin, TX-78701, USA \\ E-mail: alu@mail.utexas.edu
}

Received 24 September 2015, revised 2 December 2015

Accepted for publication 3 December 2015

Published DD MM 2016

\begin{abstract}
The powerful tools of transformation optics (TO) allow an effective distortion of a region of space by carefully engineering the material inhomogeneity and anisotropy, and have been successfully applied in recent years to control electromagnetic fields in many different scenarios, e.g., to realize invisibility cloaks and planar lenses. For various field transformations, it is not necessary to use volumetric inhomogeneous materials, and suitably designed ultrathin metasurfaces with tailored spatial or spectral responses may be able to realize similar functionalities within smaller footprints and more robust mechanisms. Here, inspired by the concept of metamaterial TO lenses, we discuss field transformations enabled by parity-time (PT) symmetric metasurfaces, which can emulate negative refraction. We first analyze a simple realization based on homogeneous and local metasurfaces to achieve negative refraction and imaging, and we then extend our results to arbitrary PT-symmetric two-port networks to realize aberration-free planar imaging.
\end{abstract}

Keywords: metamaterials, parity-time symmetry, transformation optics

(Some figures may appear in colour only in the online journal)

\section{Introduction}

The control of the electromagnetic properties of materials in a specific frequency range of operation is a challenging problem that has puzzled scientists in a variety of disciplines for many decades. With the advent of metamaterials [1, 2], in which suitably designed subwavelength inclusions are arranged in a host medium to obtain a controllable effective response, the problem of designing materials with the desired electromagnetic features was solved under certain (sometimes restrictive) constraints. Such limitations are imposed by the resonant nature of the inclusions in the considered portion of the spectrum, intrinsically associated with a large level of material loss, as well as by homogenization imperfections and fabrication issues. However, despite these challenges, the metamaterial paradigm has without doubt opened an entirely

\footnotetext{
${ }^{3}$ These authors contributed equally to the work.
}

new landscape of possibilities to control the electromagnetic wavefront and, accordingly, mold the interacting fields at will.

The idea of manipulating fields with metamaterials was further generalized and set on solid foundations with the introduction of transformation optics (TO) concepts [3], which constitute an elegant mathematical tool to realize an effective distortion of space (and, thereby, of the light path) by using materials with suitable inhomogeneous and anisotropic optical properties. This analytical concept has been extensively applied to design cloaks $[4,5]$ and lenses [6-8], and it may be used to engineer a large number of wavesteering electromagnetic devices with arbitrarily tailored response. In this context, the idea of 'imaging', namely, the exact reproduction of a source distribution from an original 'object space', to another 'image space', represents a powerful demonstration of field transformations. Lenses, in fact, possess a pivotal position within the set of devices that control electromagnetic waves [9]. From the experimental point of view, several attempts have been recently made towards 
the realization of the proposed TO designs for lenses (or other structures) by exploiting polymer photonic crystals [10], properly processed silicon-based dielectrics [11, 12], graphene [13], among several other implementations.

In this work, we review our recent work, and further explore in detail the design of planarized metasurface lenses that are transversely homogeneous. These properties ensure that the proposed lenses work in exactly the same way regardless of the transverse position of the source object, different from curved or transversely graded lenses, for which the source should be located on (or near) the symmetry axis of the device to avoid image aberrations. Furthermore, planar transversely invariant lenses are afocal, namely they have infinite focal length due to the zero curvature of its shape (or phase profile) and, thereby, they follow different imaging properties compared to conventional imaging systems. These properties are highly beneficial to achieve aberration-free imaging on more than one transverse plane, which is theoretically impossible with lenses of finite focal length, as initially shown by Maxwell [14] and later elaborated in a number of studies $[9,15,16]$. In addition, only recently planar lenses have attracted significant attention, mainly because they are not directly affine to the human vision system, which is based on the curvilinear shape of the eyeball. Therefore, while for centuries large efforts have been devoted to remedy aberrations in spherically-shaped lenses [15-18], only a few studies have been dedicated to planar configurations.

The most notable attempt to develop a planar lens has been done in the early 2000s by exploiting the unusual properties of double negative (DNG) media. In particular, it was found that a matched DNG metamaterial slab makes a perfect lens, which creates perfect aberration-free images of arbitrary 2D source distributions on any transverse plane [19]. This pioneering idea is indeed one of the foundational, and most relevant implementations of TO, since it uses DNG media to emulate a negative space or, in other words, a space that folds over itself, so that the source field is replicated (imaged) on each of the folds. Interestingly, as we have recently envisioned in [20,21], and as we further elaborate in the following, a PT-symmetric pair of metasurfaces can realize a similar response for propagating waves, without requiring $\mathrm{DNG}$ materials.

A DNG lens exploits the negative refraction of propagating waves as they enter the DNG medium, as well as the amplification of evanescent waves, to produce perfect $2 \mathrm{D}$ images whose transverse sharpness is not bounded by the resolution limit imposed by the wavelength of light [9, 15]. However, this perfect result is accompanied by poor performance of the lens in the longitudinal direction. In fact, the amplification of evanescent waves in the DNG slab determines strong resonant fields at the exit interface of the slab, which inevitably distort the image in the longitudinal direction. In other words, given a 3D region of space, a DNG lens cannot produce a perfect 3D image, but can operate ideally only over a given transverse plane provided that the source object is limited to the corresponding plane of interest. In addition, the operation of such a lens becomes sensitive to the unavoidable losses of DNG materials, as well as to granularity and imperfections. Therefore, the interesting problem of designing a close-to-perfect planar lens with robust 3D imaging performance (across both transversal and longitudinal directions) remains open.

Here, we explore a device that achieves this goal based on the concept of parity time (PT) symmetry. This intriguing concept stems from the realm of quantum mechanics, associated with Hamiltonians that are invariant after a combined time-reversal and a parity operation, which have been shown to be able to support real eigenfrequencies despite being nonHermitian [22]. Analogously, PT-symmetric electromagnetic systems are characterized by balanced loss and gain, which allows supporting non-decaying modal propagation despite having regions with loss [23-31]. PT-symmetric structures are therefore characterized by two mirrored regions, one of which is passive and the other one is equally active, so that the response of the system is identical under reversal of space and time. Recently, the application of PT-symmetry concepts to electromagnetic configurations has unveiled interesting properties and functionalities, from unidirectional cloaking $[27,31]$ to loss-immune negative refraction [20, 21], among many others.

Since negative refraction is the necessary ingredient leading to 2D planar imaging in the case of a DNG slab [19], we first consider a PT-symmetric structure similar to the one introduced in [20] in order to emulate such response. We first show that the use of local ultrathin transversely homogeneous metasurfaces as in [20] leads to a non-ideal imaging performance, and we derive the optimal configuration to achieve the best planar imaging using local, homogeneous, PT symmetric surfaces. Then, we consider more general PT-symmetric systems, described by their scattering and transfer matrices, and we derive the most general conditions for realizing ideal planar imaging, reviewing and extending our recent theoretical results reported in [21]. A few numerical examples will illustrate the operation of the proposed PT-symmetric lenses. Due to their generality, the obtained results may inspire further experimental and theoretical efforts in controlling and transforming electromagnetic fields using the power and flexibility of PT-symmetric systems.

\section{Local PT-symmetric metasurfaces}

We start by considering the simplest PT-symmetric structure that imitates a DNG lens [19], based on two parallel planar impedance sheets, or metasurfaces, one passive and the other equally active, as originally studied in [20]. The simplicity of the structure is due to the zero thickness of the impedance sheets and their transversely homogeneous nature and local response. For the functionality of interest to be achieved, as discussed extensively in [20], the passive component of the system (with surface impedance $+Z$ ) should act as a coherent perfect absorber (CPA) [34], namely, as a two-port network that absorbs all the incoming energy only when it is excited bilaterally with waves respecting a certain phase relation. The active component (impedance $-Z$, time-reversed of the passive surface) is the PT-symmetric counterpart of the CPA, 


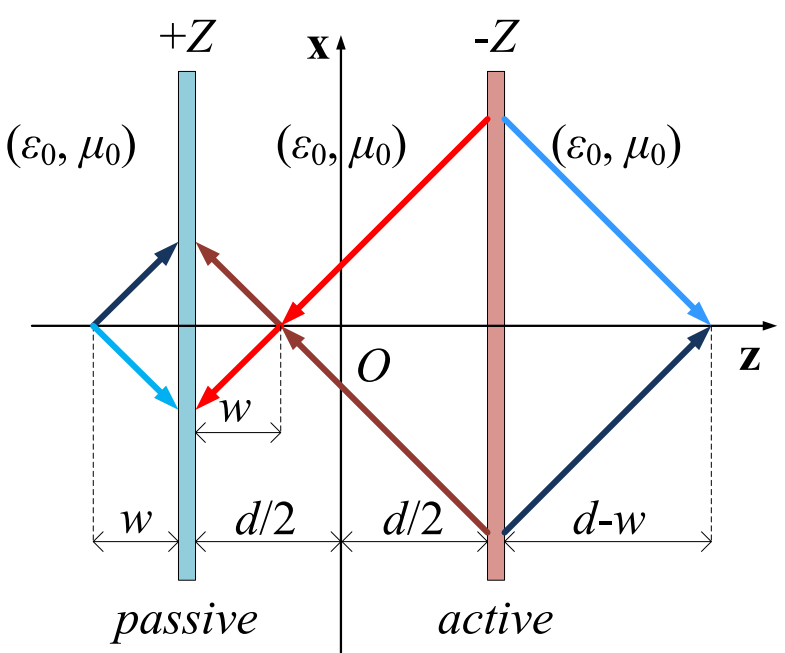

(a)

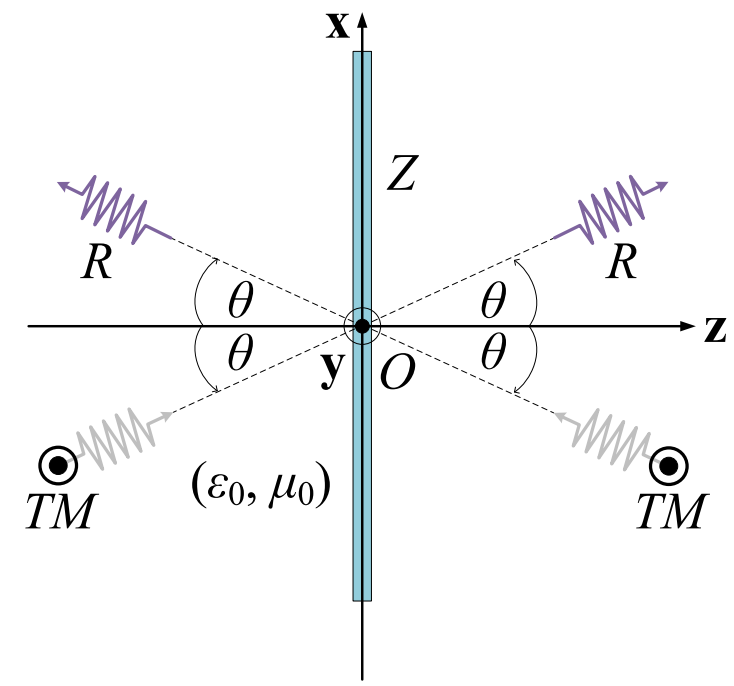

(b)

Figure 1. (a) PT-symmetric configuration composed of a pair of metasurfaces separated by a distance $d$. The system is excited by a line source located at distance $w$ from the passive element. At steady state, the active metasurface produces the required waves to make the passive metasurface work as a CPA, creating backward phase and power flow between the two elements, and producing an image of the source at a distance $(d-w)$ from the active element. (b) Configuration of a single metasurface excited bilaterally by TM-polarized plane waves propagating along directions forming angles $\pm \theta$ with the horizontal $\mathbf{z}$ axis.

namely, a lasing structure that emits coherently on both sides with the same phase relation. This principle of operation is shown in figure 1(a), where the distance between the two planar impedance sheets is $d$ and optical rays are emitted by a line source at distance $w$ from the passive metasurface. At steady state, the active metasurface coherently emits waves on both sides, synchronized with the source: the waves emitted towards the left converge towards a point (internal image) and get absorbed by the passive metasurface by properly interfering with the field radiated by the source, meeting the CPA requirement on bilateral excitation, while the waves emitted towards the right of the active surface form an external image at distance $(d-w)$. It is important to stress that transmission of information and synchronization between object plane and image plane occur, on their own and without external control, during the transient regime. The two planes appear fully decoupled in steady state, due to the perfect absorption occurring at the left element. On the contrary, as the source is turned on, or after any change in the spectrum of the source field, the excitation of the left element is found unbalanced, and therefore the CPA functionality allows partial transmission of the input signal towards the active element, which can synchronize in this transient regime with the source.

After any modification of the incident field distribution, it takes a few cycles before the transmitted image is updated and the CPA can receive the required balanced excitation from the two sides to absorb again the entire input fields. This transient response depends on the bandwidth of operation of the device, which is fundamentally limited only by the complexity of the dispersion of the passive and active elements, and by the requirements on stability. In practical situations, the time required to reach steady-state is in any case expected to be quite fast from the point of view of human perception and digital image processing, hence the system would act as a real-time imaging device for all practical purposes. Related to the temporal evolution of the system in the transient domain, the stability of the system is an important issue to be considered, since it involves active elements. In [35], a related system was studied for different purposes, and it was found that it is always possible to design a specific dispersion of the active and passive elements that ensure full stability. We therefore expect that, also for the PT-symmetric lenses discussed in this paper, full stability for any finite input signal can be achieved. We will discuss the stability and temporal dispersion issues of this imaging device in a future publication.

Since the active sheet is designed to be the PT-symmetric counterpart of the passive sheet, the performance of the whole system as a focusing TO-inspired lens in steady-state is essentially determined by how well the passive metasurface acts as a CPA. In figure 1(b), we show a passive sheet of surface impedance $Z$, excited from both sides by two symmetric plane waves propagating along directions forming angles $\pm \theta$ with the horizontal axis $\hat{\mathbf{z}}$. Without loss of generality, we consider TM waves with incident magnetic field $\mathbf{H}=\hat{\mathbf{y}} \mathrm{e}^{-j k_{x} x \pm j k_{0} z \cos \theta}=\hat{\mathbf{y}} \mathrm{e}^{-j k_{0}(x \sin \theta \pm z \cos \theta)} \quad$ where $k_{0}=2 \pi f \sqrt{\varepsilon_{0} \mu_{0}}$ is the free-space wavenumber $\left(\varepsilon_{0}\right.$ is the dielectric permittivity and $\mu_{0}$ the magnetic permeability of freespace, under an $\mathrm{e}^{+j \omega t}$ harmonic time dependence). The reflection coefficient characterizing the two outgoing waves is denoted by $R$, while the $\hat{\mathbf{x}}$-directed wavenumber is written as: $k_{x}=k_{0} \sin \theta$. For now we assume that the surface impedances in figure 1 are local, namely, their response does not depend on the wavevector of the impinging wave, a feature that, as we will see in the following, prevents the device from acting ideally in terms of imaging, since it cannot compensate 


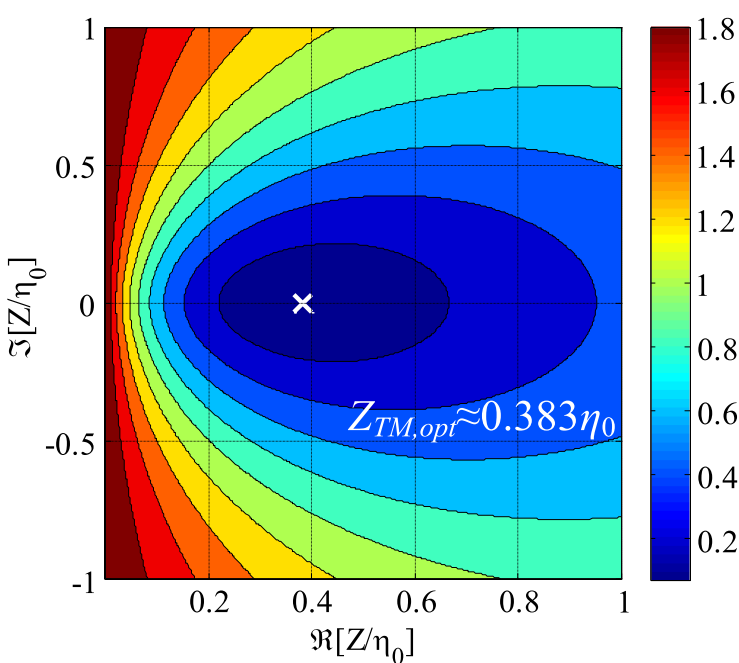

(a)

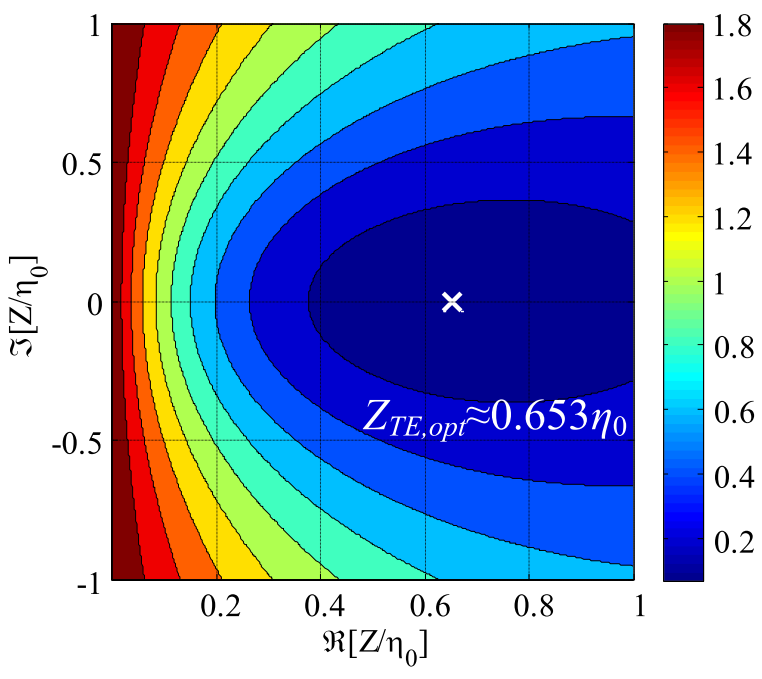

(b)

Figure 2. Figure of merit $r$, defined by integrating the reflected power over all incidence angles as in equation (1), as a function of the real and imaginary parts of the metasurface impedance $Z$ normalized to the free-space wave impedance $\eta_{0}$ for: (a) TM case and (b) TE case. The white crosses denote the optimal choices for minimum reflections $r$. The numerical values are given by equation (2).

the natural angular dispersion of the wave impedance in freespace. A suitable metric $r$ to quantify the performance of the passive impedance sheet acting as a bilateral absorber (and, accordingly, indicating the focusing performance of the entire PT-symmetric system) should take into account the reflected power from waves spanning the entire propagating wavenumber spectrum, i.e., waves with $\left|k_{x}\right|<k_{0}$ :

$$
r=\int_{-k_{0}}^{k_{0}}\left|R\left(k_{x}\right)\right|^{2} \mathrm{~d} k_{x}=\int_{-\pi / 2}^{\pi / 2}|R(\theta)|^{2} \cos \theta \mathrm{d} \theta .
$$

Here, we do not consider the contribution of evanescent modes, because they are not time-reversible and they would lead to exponentially diverging fields if they would contribute to the imaging functionality, as discussed in the introduction. In figure 2, we show the variation of $r$ with respect to the real and the imaginary parts of surface impedance $Z$ for both TM (figure 2(a)) and TE (figure 2(b)) polarizations, assuming bilateral symmetric excitation as shown in figure 1(b). The minimum reflected power, integrated over all incidence angles $\theta$ as in (1), is the same for both cases $\left(r_{\min } \cong 0.688\right)$. The white crosses show the optimal choices for the surface impedance $Z$, corresponding to the values:

$$
Z_{\mathrm{TM}, \mathrm{opt}} \cong 0.383 \eta_{0} \quad Z_{T \mathrm{E}, \mathrm{opt}} \cong 0.653 \eta_{0},
$$

where $\eta_{0}=120 \pi \Omega$ is the free-space wave impedance. Interestingly, both optimal impedances are real, and they are related by the relation: $Z_{\mathrm{TM}, \mathrm{opt}} Z_{T \mathrm{E} \text {, opt }}=\left(\frac{\eta_{0}}{2}\right)^{2}$. Instead, the impedance value $\frac{\eta_{0}}{2}$, considered in [20], corresponds (for both polarizations) to zero reflection for normal incidence $(\theta=0)$, but leads to higher overall reflection for other angles, according to equation (1) and figure 2.

As discussed in the introduction, our goal is to achieve aberration-free ideal imaging in the sense of geometrical optics, meaning that the field distribution of the image of an arbitrary line source is approximated by its entire propagating spectrum. Therefore, the incident magnetic field that we aim to focus, defined in $\left(z<-L-\frac{d}{2}\right)$, is not a Hankel function $H_{0}^{(2)}\left(k_{0} \sqrt{x^{2}+\left(z+L+\frac{d}{2}+w\right)^{2}}\right)$, but its truncated spectral representation with respect to $k_{x}$ limited by diffraction $\left(\left|k_{x}\right|<k_{0}\right)$; namely [32, 33]

$$
\begin{aligned}
H_{y, \text { inc }}(x, z) & =\frac{j}{\pi} \int_{-k_{0}}^{k_{0}} \frac{\mathrm{e}^{-j\left|z+L+\frac{d}{2}+w\right| \sqrt{k_{x}^{2}-k_{0}^{2}}}}{\sqrt{k_{x}^{2}-k_{0}^{2}}} \mathrm{e}^{-j k_{x} x} \mathrm{~d} k_{x} \\
= & J_{0}\left(k_{0} \sqrt{x^{2}+\left(z+L+\frac{d}{2}+w\right)^{2}}\right)
\end{aligned}
$$

where $J_{0}$ denotes the 0th-ordered Bessel function, and $L$ denotes the thickness of the focusing elements. In other words, the best focusing result at the image position, $z_{i}=L+\frac{d}{2}+d-w$, that one can possibly achieve with the proposed PT-symmetric structure is given by a field at $z_{i}$ given by (3).

In figure 3, we show the calculated spatial distribution (time snapshot) of the magnetic field $(\mathbf{H} \cdot \hat{\mathbf{y}})$ for two TM excitations. In figure 3(a), the impedance of the passive metasurface (with zero thickness $L=0$ ) has been chosen to realize zero reflection at normal incidence, i.e., $Z=0.5 \eta_{0}$, corresponding to the case studied in [20], whereas in figure 3(b) the optimal impedance has been selected according to figure 2 , i.e., $Z \cong 0.383 \eta_{0}$. It is clear that the focusing performance in the latter case is improved, as the focal spots are stronger and narrower. We have explored several different realizations of local systems that may synthesize this functionality, even relaxing the assumption of ultrathin metasurfaces, and our study, consistent with figure 2, indicates that the best imaging performance that can be achieved with a PTsymmetric lens composed of local elements is provided by the 


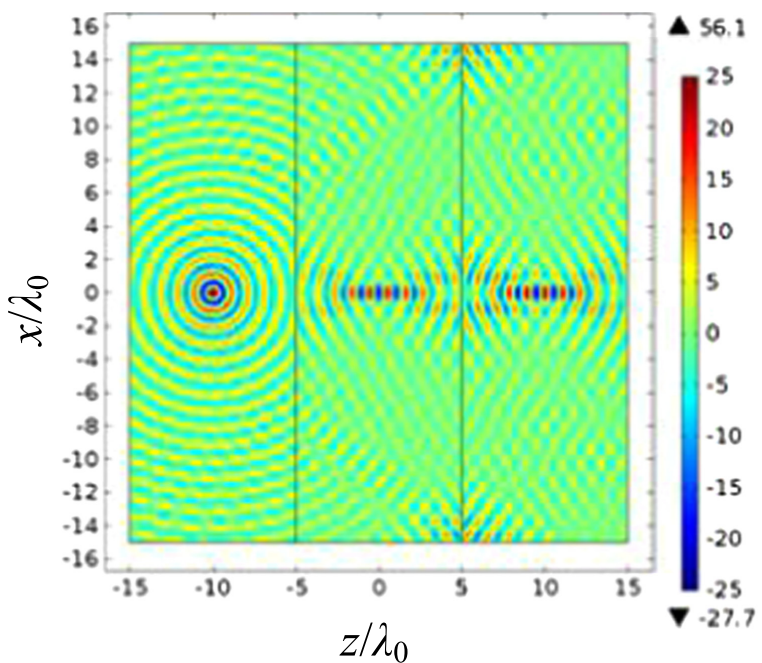

(a)

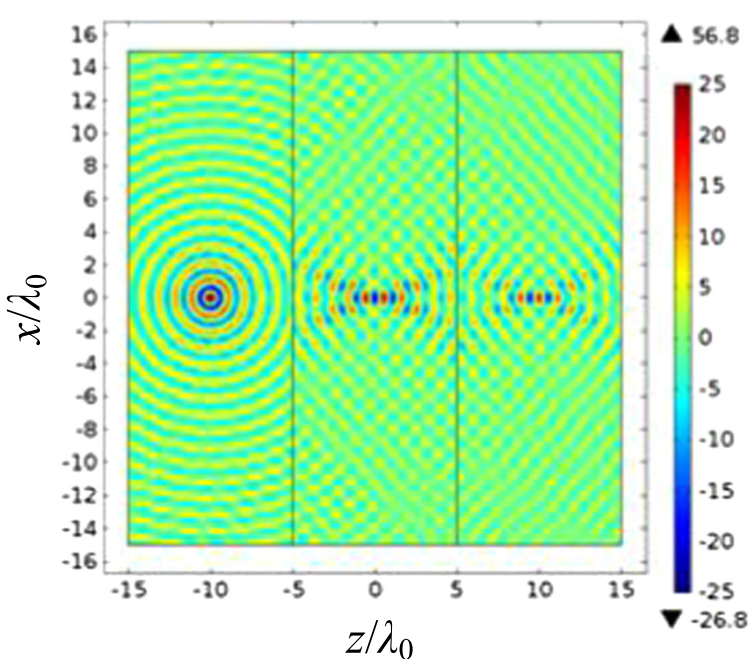

(b)

Figure 3. Spatial distribution (time snapshot) of the magnetic field $(\mathbf{H} \cdot \hat{\mathbf{y}})$ when a pair of local PT-symmetric metasurfaces is excited by a TM-polarized line source for: (a) $Z=0.5 \eta_{0}$ as in [20], and (b) $Z=Z_{\mathrm{TM} \text {,opt }} \cong 0.383 \eta_{0}$, corresponding to the optimal impedance in figure 2(a). Plot parameters: $d=10 \lambda_{0}, w=5 \lambda_{0}=d / 2$.

result in figure 3(b). It is evident, however, that the obtained configuration is far from being an ideal aberration-free lens. Therefore, in the next section, we will investigate the problem of perfect imaging from a more general viewpoint, which will allow designing ideal PT-symmetric lenses and other fieldmanipulating devices.

\section{PT-symmetric systems for transformation-optics- inspired imaging}

Relaxing the assumption that the transversely homogeneous elements of the proposed PT-symmetric system have to be ultrathin and local, as originally envisioned in [21], we show in the following that a similar functionality as the one described in the previous section may be achieved by an arbitrary pair of PT-symmetric systems, one of which acts as a CPA. In the most general scenario, a generic two-port network described by the scattering matrix $\mathbf{S}=\left[\begin{array}{ll}S_{11}(\theta) & S_{12}(\theta) \\ S_{21}(\theta) & S_{22}(\theta)\end{array}\right]$ can realize a generalized CPA $(r=0)$, which absorbs all the impinging energy when bilaterally excited with equal power and arbitrary phase difference $\xi \in[0,2 \pi)$, if and only if $\mathbf{S}$ is written in the following general form:

$$
\mathbf{S}=\left[\begin{array}{cc}
-a \mathrm{e}^{j \xi} & a \\
b & -b \mathrm{e}^{-j \xi}
\end{array}\right]
$$

$$
\mathbf{T}=\frac{1}{2 b}\left[\begin{array}{c}
1-\mathrm{e}^{j \xi}(a-b+2 j a b \sin \xi) \\
\frac{1+\mathrm{e}^{j \xi}(a+b+2 j a b \sin \xi)}{Z_{0}}
\end{array}\right.
$$

where $a, b \in \mathbb{C}$ can be any complex number regardless of their magnitudes, and in general they can be a function of the

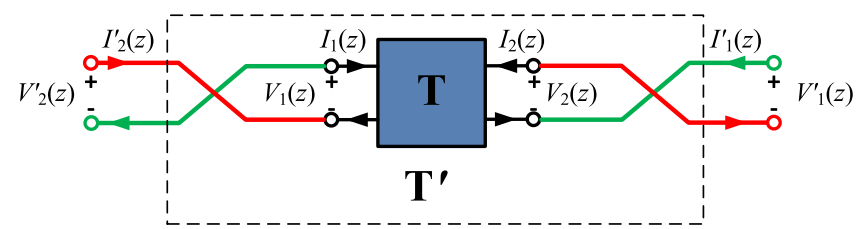

Figure 4. Sketch of a generic transfer matrix $\mathbf{T}$ and its PT-symmetric counterpart $\mathbf{T}^{\prime}$ obtained by twisting and flipping the two ports of the original network.

angle $\theta$. This is simply obtained by requiring that, for excitation with same amplitude and phase difference $\xi$ from the two sides, the outgoing waves are identically zero. Note that, in general, this structure is not required to be symmetric or reciprocal and, in addition, a coherent absorber is not necessarily passive, as we can have $\left|S_{11}\right|^{2}+\left|S_{12}\right|^{2}>1$. It is also relevant to briefly point out the difference between this CPA functionality, which requires interference between reflected and transmitted waves from both ports [34], and other common absorbers (see, e.g., [36]), which are unilaterally excited and aim at achieving maximal attenuation of a single incident beam.

As it will be clear in a moment, it is convenient to work with the associated transfer matrix of the generalized CPA in equation (4), given by

$$
\left.\begin{array}{c}
Z_{0}\left[1-\mathrm{e}^{j \xi}(a+b-2 j a b \sin \xi)\right] \\
1+\mathrm{e}^{j \xi}(a-b-2 j a b \sin \xi)
\end{array}\right],
$$

where $Z_{0}=\eta_{0} \cos \theta$ is the TM wave impedance, function of the incidence angle $\theta$. In order to obtain the PT-symmetric 


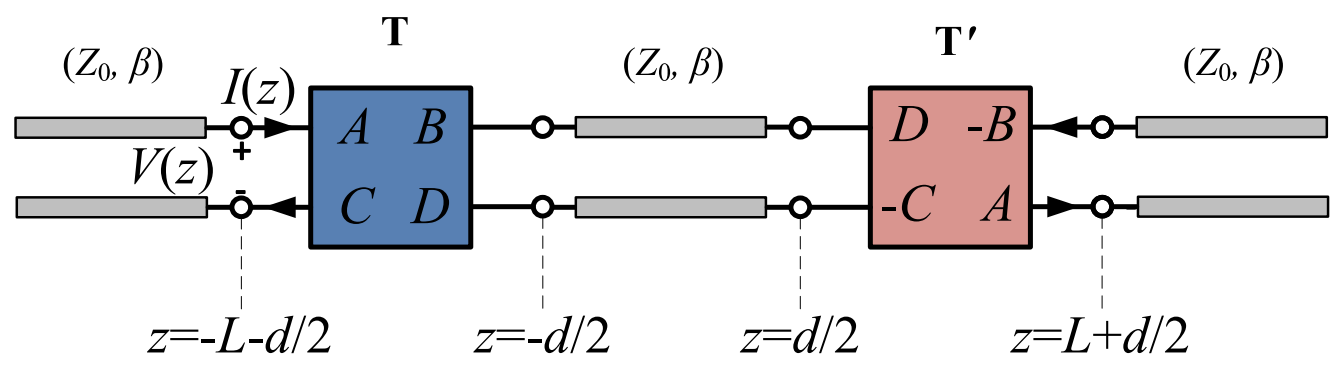

Figure 5. Transmission-line representation of the overall system. The absorber with transfer matrix $\mathbf{T}$ and thickness $L$ is separated from its PT-symmetric counterpart (with transfer matrix $\mathbf{T}^{\prime}$ and the same thickness $L$ ) by an airgap of length $d$. The wavenumber $\beta=k_{0} \cos \theta$ and the wave impedance $Z_{0}=\eta_{0} \cos \theta$ correspond to obliquely incident TM waves.

element of this one, we notice, according to the sketch in figure 4, that for arbitrary $\mathbf{T}=\left[\begin{array}{ll}A & B \\ C & D\end{array}\right]$, its PT-symmetric counterpart has the transfer matrix

$$
\mathbf{T}^{\prime}=\frac{1}{A D-B C}\left[\begin{array}{cc}
D & -B \\
-C & A
\end{array}\right]
$$

In fact, the twist and the flip of the ports in figure 4 realizes both parity and time reversal. Equation (6) corresponds to the operation of taking the inverse of the original transfer matrix. Therefore, the transfer matrix of the PT-symmetric counterpart of (5) reads

$$
\begin{aligned}
& \mathbf{T}^{\prime}= \\
& \frac{1}{2 a}\left[\begin{array}{cc}
1+\mathrm{e}^{-j \xi} a-\mathrm{e}^{j \xi} b & -Z_{0}\left(1-\mathrm{e}^{-j \xi} a-\mathrm{e}^{j \xi} b\right) \\
-\frac{1}{Z_{0}}\left(1+\mathrm{e}^{-j \xi} a+\mathrm{e}^{j \xi} b\right) & 1-\mathrm{e}^{-j \xi} a+\mathrm{e}^{j \xi} b
\end{array}\right] \text { (7) }
\end{aligned}
$$

Interestingly, the corresponding scattering matrix $\mathbf{S}^{\prime}$ diverges, since it describes an ideal lasing system.

If we now consider the entire PT-symmetric system, comprised of the absorbing component (with transfer matrix $\mathbf{T}$ and thickness $L$ ) and its time-reversed counterpart (with transfer matrix $\mathbf{T}^{\prime}$ and same thickness), positioned in freespace at a distance $d$ from each other, we obtain the system shown in figure 5. The vacuum is modeled as a transmission line with wavenumber $k_{y}=k_{0} \cos \theta$ and TM characteristic impedance $Z_{0}=\eta_{0} \cos \theta$. The transfer matrix of the entire system can be readily obtained as the cascade (matrix multiplication) of the different blocks [37]. The corresponding scattering matrix is then given by

$$
\begin{aligned}
S^{\text {total }} & =\left[\begin{array}{cc}
S_{11}^{\text {total }}(\theta) & S_{12}^{\text {total }}(\theta) \\
S_{21}^{\text {total }}(\theta) & S_{22}^{\text {total }}(\theta)
\end{array}\right] \\
& =\left[\begin{array}{cc}
0 & \mathrm{e}^{j k_{0} d \cos \theta+2 j \xi} \\
\mathrm{e}^{j k_{0} d \cos \theta+2 j \xi} & \frac{\mathrm{e}^{2 j k_{0} d \cos \theta+2 j \xi}-1}{a} \mathrm{e}^{j \xi}
\end{array}\right] .
\end{aligned}
$$

This matrix describes a very interesting optical response, with several implications for imaging and wave transformation purposes. First of all, the response is independent of the thickness $L$ of the two sub-systems (CPA and laser). The device exhibits zero reflection from the CPA side and it realizes negative refraction like a DNG lens of thickness $d$ with an additional phase shift $2 \xi$, remarkably independent of the parameters $a$ and $b$, as well as of their dependence on the angle of incidence. In other words, such PT-symmetric system, independent of the specific amplitude response of choice, phase advances the transmitted wave by $\left(k_{0} d \cos \theta+2 \xi\right)$ for all incidence angles. The overall system is reciprocal $\left(S_{12}^{\text {total }}(\theta)=S_{21}^{\text {total }}(\theta)\right)$ even though the CPA and lasing elements may individually be non-reciprocal, i.e., $a \neq b$, since equation (7) does not depend at all on the complex parameter $b$.

The field distributions for line source excitation can be found using a conventional plane-wave expansion. It is simple to prove that the magnetic field transmitted through the system (8), at $z_{i}=L+\frac{d}{2}+d-w$, equals

$$
H_{y}\left(x, z=z_{i}\right)=J_{0}\left(k_{0} x\right) \mathrm{e}^{j 2 \xi}
$$

for a line source excitation. Therefore, consistent with equation (3), such a device works as an aberration-free planar lens for all propagating angles $-\frac{\pi}{2}<\theta<\frac{\pi}{2}$, namely, it produces an image identical to the source, in amplitude and phase, for $\xi=0, \pi$. As pointed out above, this property is not affected by the value of the complex parameters $a$ and $b$ in (4), (8) because the transmission coefficient $S_{21}^{\text {total }}(\theta)=\mathrm{e}^{j k_{0} d \cos \theta+2 j \xi}=S_{12}^{\text {total }}(\theta)$ does not depend on them. Even though $a$ and $b$ are angle dependent, as long as their dependence respects the PT-symmetry condition, the functionality is still ideally preserved. These observations allow identifying a broad class of systems able to realize ideal imaging in the sense of geometrical optics, the only conditions being CPA operation and PT symmetry, which is consistent with, and generalizes, the findings in [21]. In particular, these conditions generally correspond to having a nonlocal (or spatially dispersive) response, as the structure compensates the natural dispersion of the wave impedance as a function of angle. Such an effective nonlocal response can be implemented, for example, by using thin multilayered slabs, as discussed in [21], also in analogy with the computational metastructures proposed in [38].

As an example of ideal imaging, in figure 6 we show the variation of the real and the imaginary parts of the magnetic field $H_{y}=(\mathbf{H} \cdot \hat{\mathbf{y}})$ for two PT-symmetric lenses illuminated by a TM-polarized line source; the first row corresponds to a lens designed to have zero phase shift, $\xi=0$, and the second row for the case with $\xi=\pi$. In both cases, the field is focused around the axis: $(x, z)=\left(0, \frac{3 d}{2}+L-w\right)$ at the right of the 


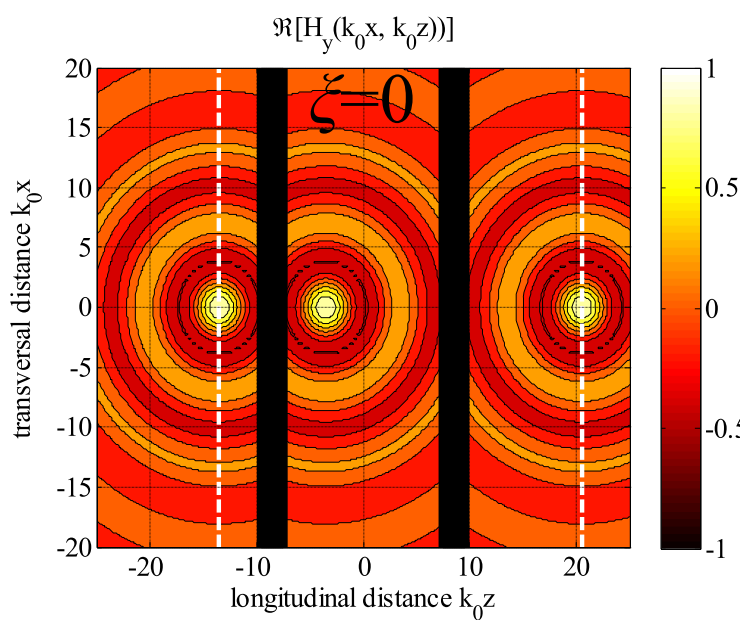

(a)

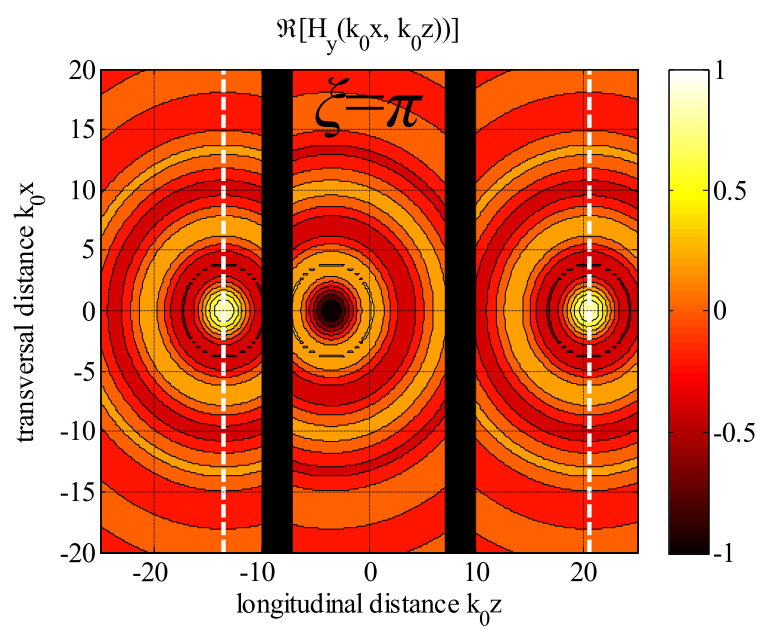

(c)

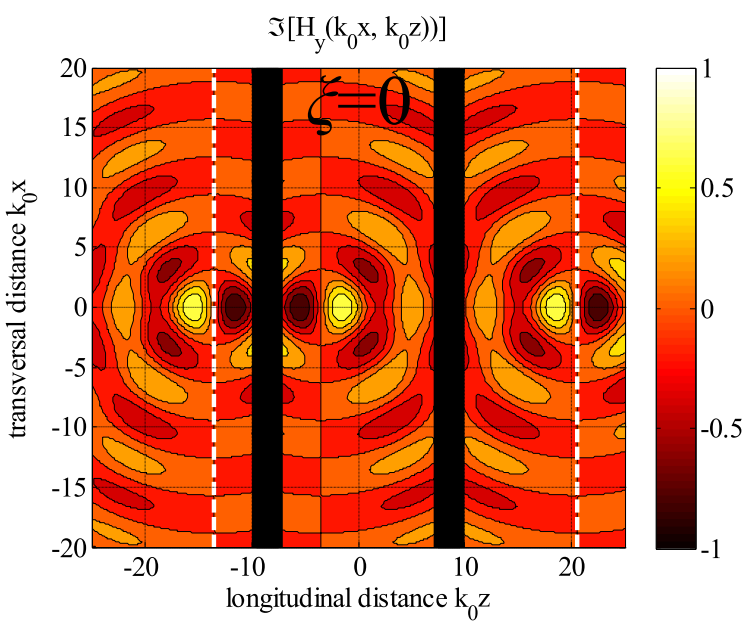

(b)

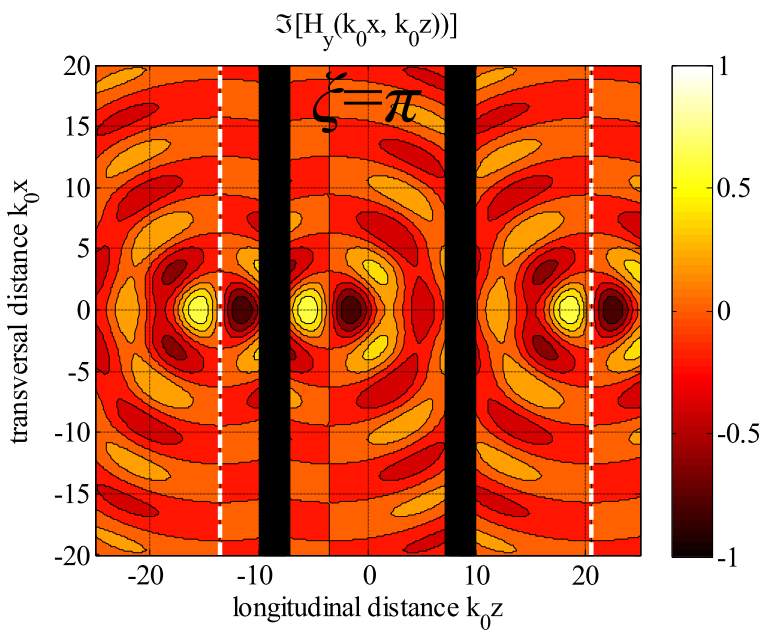

(d)

Figure 6. Spatial distribution of the magnetic field $(\mathbf{H} \cdot \hat{\mathbf{y}})=H_{y}$ for TM line-source illumination, when the CPA has been designed for phase shift $\xi=0$ ((a) and (b)) and $\xi=\pi((\mathrm{c})$ and (d)). The real part is shown in subfigures (a) and (c), and the imaginary part in subfigures ((b) and (d)). Plot parameters: $k_{0} L=2, k_{0} d=15, k_{0} w=4$.

active element; in addition, an internal image forms between the two PT-symmetric elements of the lens (of finite thickness $L$ depicted as black boxes) around the axis: $(x, z)=\left(0,-\frac{d}{2}+w\right)$. The white dashed lines correspond to the image and the source planes. Note that the field distribution outside the two elements, namely, for $z \in\left(-\infty,-L-\frac{d}{2}\right) \cup\left(L+\frac{d}{2},+\infty\right)$, is identical for both phase shifts, and it corresponds to the response of an ideal lens limited to propagating wave, as dictated by (3). Instead, as clearly seen in figure 6 , the phase shift $\xi=0, \pi$ affects the internal images, which are accordingly phase delayed. In fact, for TM polarization a zero phase shift $\xi=0$ corresponds to a CPA designed for in-phase bilateral excitation, while the choice $\xi=\pi$ corresponds to an out-of-phase one (opposite for TE polarization). The results reported in figure 6 show that the image produced by the PT-symmetric lens is perfect (in the sense of geometrical optics) not only along the plane $z=\frac{3 d}{2}+L-w$, but also on the entire free-space region at the right of the lens, $z>d+L$. In other words, we have indeed realized an aberration-free planar 3D lens limited by diffraction.

It is also interesting to investigate what happens for an arbitrary value of phase shift $\xi \in[0,2 \pi)$. In figure 7 , we show the real part of the magnetic field (time snapshot) on the map $\left(k_{0} x, k_{0} z\right)$ for various phase shifts. The corresponding variations of the imaginary part are given in figure 8. As predicted by equation (9), the image formed along the external focal axis, $(x, z)=\left(0, \frac{3 d}{2}+L-w\right)$, has a phase difference equal to $2 \xi$. For example, in figures $7(\mathrm{c})$ and 8 (c) perfect focusing is achieved with reversed phase, as $2 \xi=\pi$. By inspecting the left part of each graph, one can observe the operation of CPAs for various phase differences $\xi$. Similarly, the spatial distributions of the real and imaginary parts of the magnetic field around the active element of the lens are accordingly affected by the choice of $\xi$. However, it is evident from these results that the field amplitude $\left|H_{y}\left(k_{0} z, k_{0} x\right)\right|$ is identical in every single case, regardless of the selection of $\xi$. 


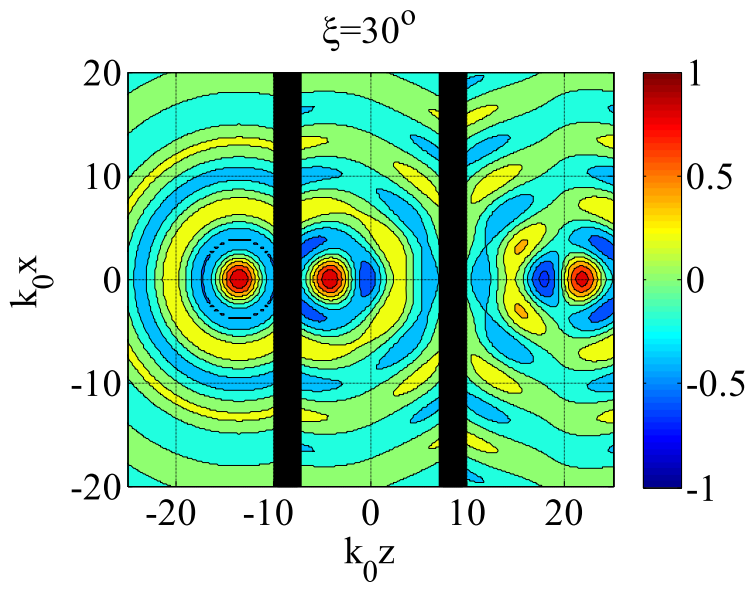

(a)

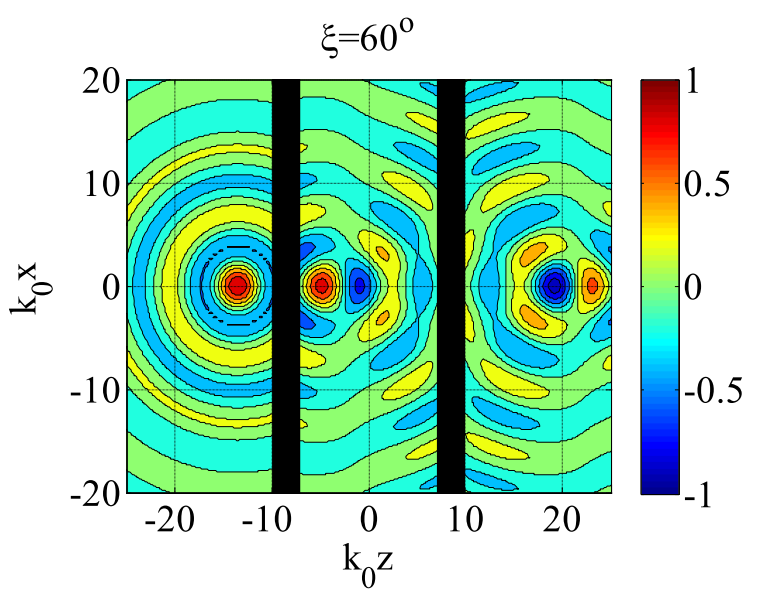

(b)

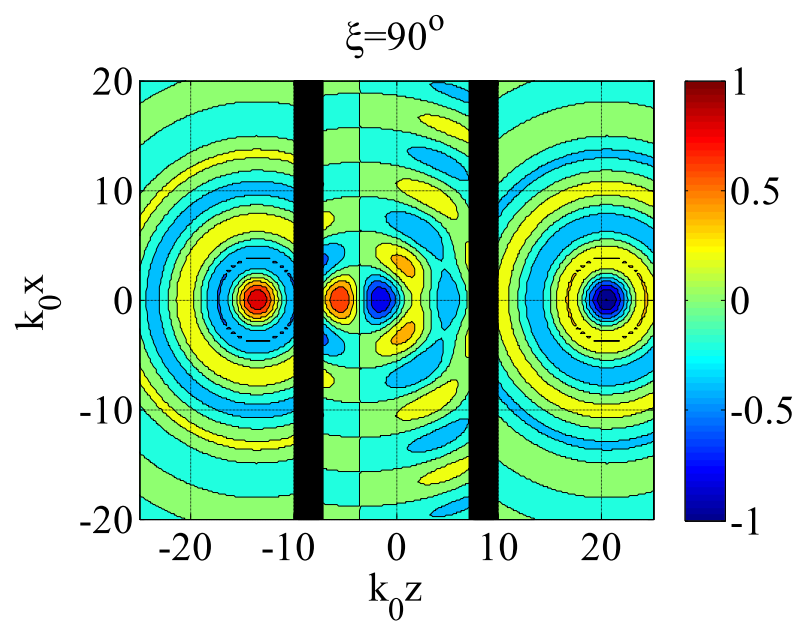

(c)

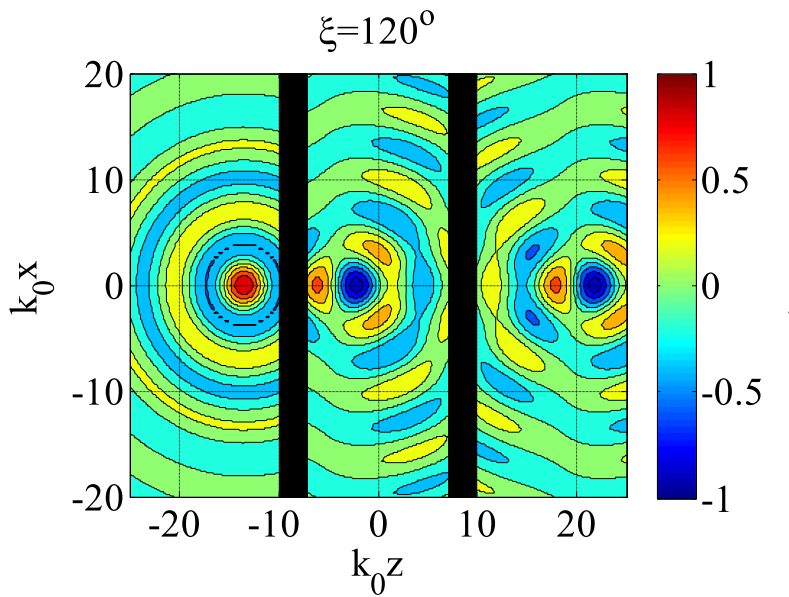

(d)

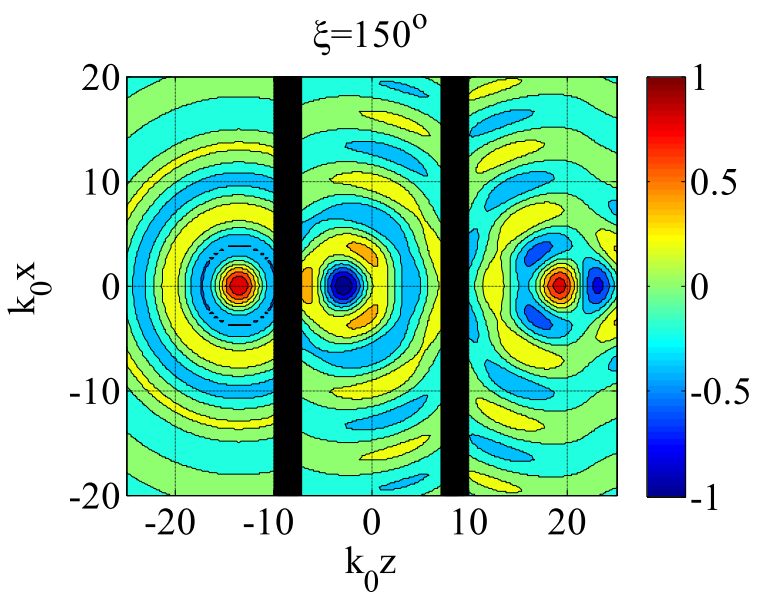

(e)

Figure 7. Spatial distribution of the real part (time snapshot) of the magnetic field $\mathfrak{R}[\mathbf{H} \cdot \hat{\mathbf{y}}]=\mathfrak{R}\left[H_{y}\right]$ for TM line-source illumination when the CPA has been designed for phase shift: (a) $\xi=30^{\circ}$, (b) $\xi=60^{\circ}$, (c) $\xi=90^{\circ}$, (d) $\xi=120^{\circ}$, (e) $\xi=150^{\circ}$. Plot parameters: $k_{0} L=2$, $k_{0} d=15, k_{0} w=4$.

To put it alternatively, ideal planar imaging (limited by diffraction), in terms of magnitude, is achieved for arbitrary angle $\xi \in[0,2 \pi)$.

The fact that an arbitrary choice of the parameter $\xi \neq 0$, $\pi$ changes the phase of the internal and external images may have interesting applications beyond imaging, for field TO. For example, a structure with varying $\xi=\xi(x)$ along the vertical axis $x$, can be used to tailor the phase of the output field at will, acting as pairs of PT-symmetric metasurfaces $[39,40]$, which would produce transformed images of the 


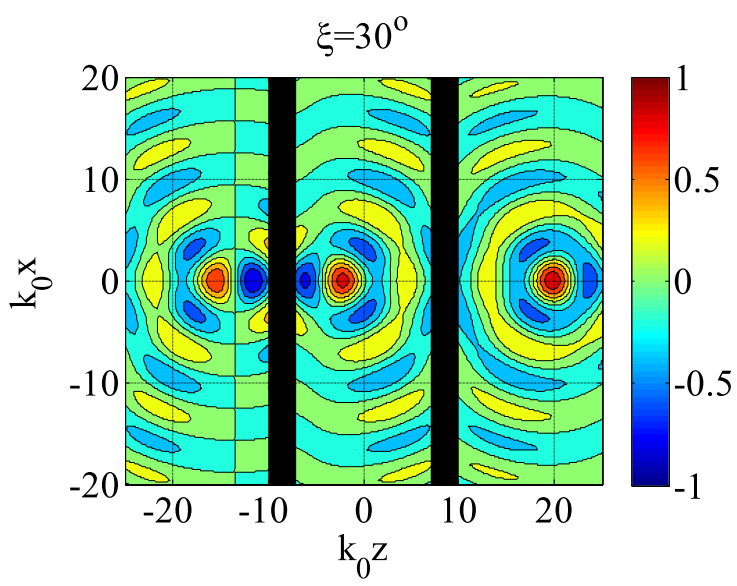

(a)

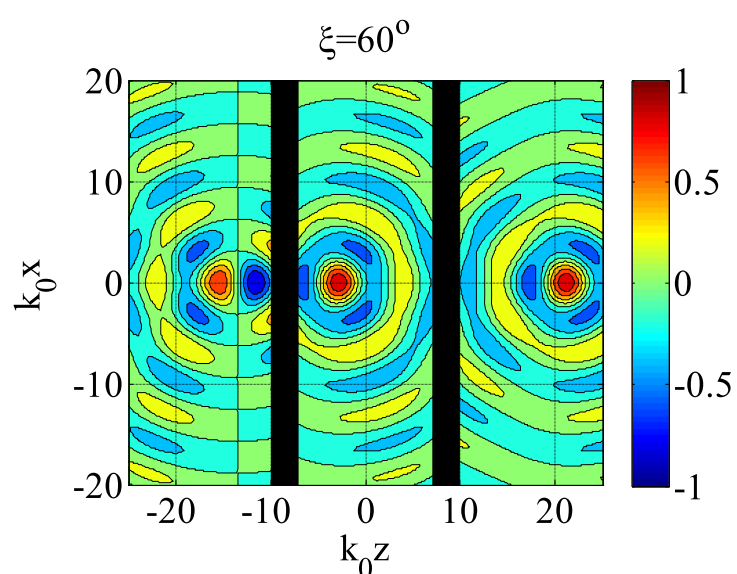

(b)

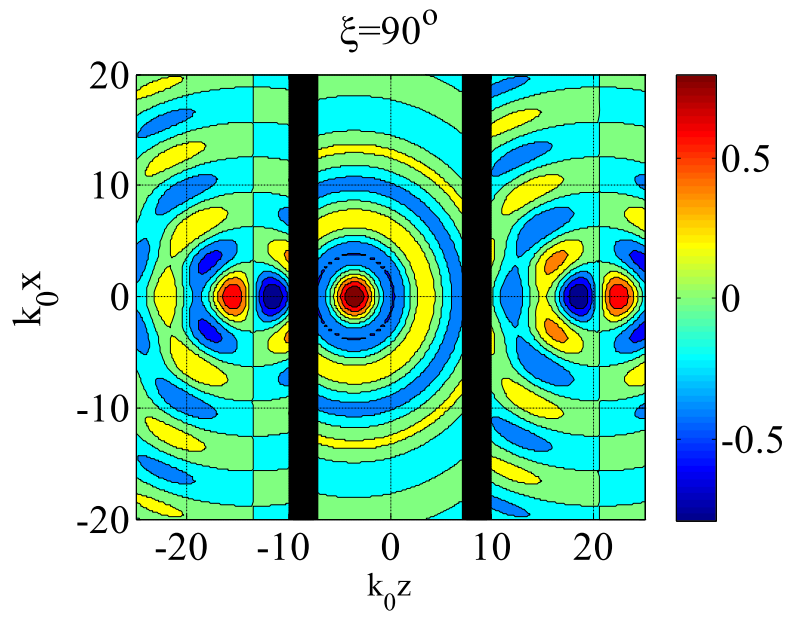

(c)

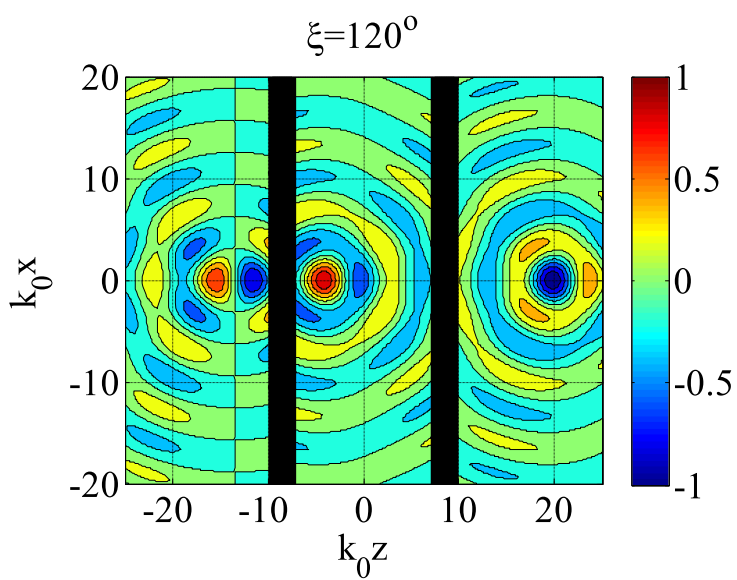

(d)

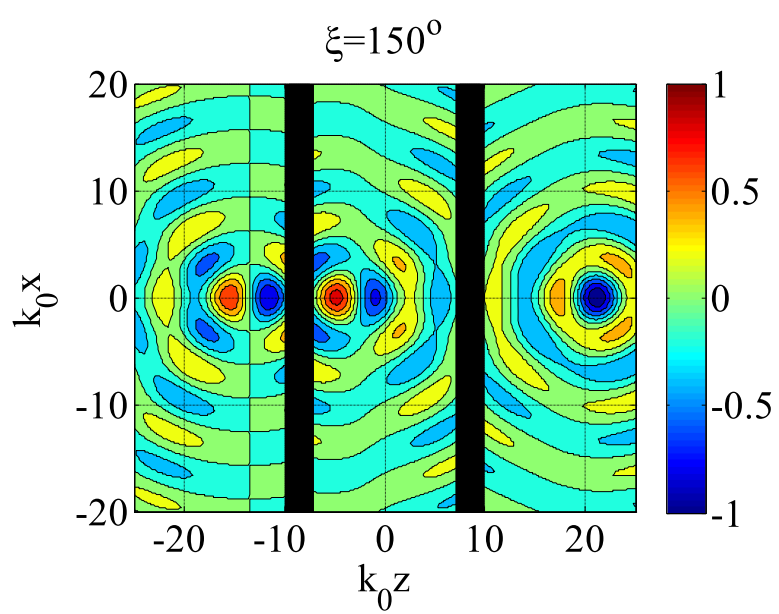

(e)

Figure 8. Spatial distribution of the imaginary part of the magnetic field $\mathfrak{I}[\mathbf{H} \cdot \hat{\mathbf{y}}]=\mathfrak{I}\left[H_{y}\right]$ for TM line-source illumination when the CPA has been designed for phase shift: (a) $\xi=30^{\circ}$, (b) $\xi=60^{\circ}$, (c) $\xi=90^{\circ}$, (d) $\xi=120^{\circ}$, (e) $\xi=150^{\circ}$. Plot parameters: $k_{0} L=2$, $k_{0} d=15, k_{0} w=4$.

source distribution. In addition, by using a suitable profile of $\xi$, these PT-symmetric systems could play the role of phase aligners for specific source distributions, maximizing their overall transmitted power by avoiding destructive interference [41]. Furthermore, since the internal images are real (different from a DNG lens for which internal images are always virtual), the designed system also allows shaping and exploiting the fields between the two PT-symmetric elements. 


\section{Conclusions}

In this paper, we have investigated the design of transversely homogeneous planar lenses and field transformations based on PT symmetry, which are able to emulate negative refraction and planar imaging in analogy with transformation-optics lenses, but based on a different mechanism, and without some of the drawbacks of conventional metamaterial implementations. In the considered PT-symmetric system, the passive component acts as a CPA, while its PT-symmetric counterpart works as a laser. We have first tested the limits of local ultrathin metasurfaces in achieving negative refraction and planar imaging. We have then identified the most general class of PT-symmetric devices that is able to realize aberration-free imaging, and we have derived the fundamental conditions to achieve this functionality, confirming and extending our previous works $[20,21]$. In conclusion, we believe that the present paper provides relevant insights into the operation of PT-symmetric systems in the context of imaging and field transformations. Our findings confirm that the path initiated by the theory of TO toward better optical systems is still very relevant today, and the concept of PT symmetry may provide new tools and possibilities to go beyond the limitations of passive systems in controlling electromagnetic fields.

This work was partially supported by the Office of Naval Research with grant No. N00014-15-1-2685 and by the Air Force Office of Scientific Research with Grant No. FA955013-1-0204.

\section{Q1 References}

[1] Pendry J B, Holden A J, Robbins D J and Stewart W J 1999 Magnetism from conductors and enhanced nonlinear phenomena IEEE Trans. Microw. Theory Tech. 47 2075-84

[2] Smith D R, Pendry J B and Wiltshire M C K 2004 Metamaterials and negative refractive index Science $\mathbf{3 0 5}$ 788-92

[3] Pendry J B, Schurig D and Smith D R 2006 Controlling electromagnetic fields Science 312 1780-2

[4] Chen H, Chan C T and Sheng P 2010 Transformation optics and metamaterials Nat. Mater. 9 387-96

[5] Leonhardt U 2006 Optical conformal mapping Science 312 1777-80

[6] Kildishev A V and Shalaev V M 2008 Engineering space for light via transformation optics Opt. Lett. 33 43-5

[7] Smith D R, Urzhumov Y, Kundtz N B and Landy N I 2010 Enhancing imaging systems using transformation optics $O p t$. Express 18

[8] Kildishev A V and Narimanov E E 2007 Impedance-matched hyperlens Opt. Lett. 32 3432-4

[9] Born M and Wolf E 1999 Principles of Optics: Electromagnetic Theory of Propagation, Interference and Diffraction of Light (Cambridge: Cambridge University Press)

Q3 [10] Wegener M 2015 Transformation Optics (Springer, NATO Science for Peace and Security Series B: Physics and Biophysics, Nano-Structures for Optics and Photonics) pp 103-8

[11] Valentine J, Li J, Zentgraf T, Bartal G and Zhang X 2009 An optical cloak made of dielectrics Nat. Mater. 8 568-71
[12] Ma H F and Cui T J 2010 Three-dimensional broadband and broad-angle transformation-optics lens Nat. Commun. 124

[13] Vakil A and Engheta N 2011 Transformation optics using graphene Science 332 1291-4

[14] Maxwell J C 1858 On the general laws of optical instruments Q. J. Pure Appl. Math. 2 271-85

[15] Smith W J 1990 Modern Optical Engineering (New York: McGraw-Hill)

[16] Smith T T 1922 Spherical aberration in thin lenses Phys. Rev. 276-7

[17] Johnson E G and Nier A O 1953 Angular aberrations in sector shaped electromagnetic lenses for focusing beams of charged particles Phys. Rev. 91 10-7

[18] Evans L R and Morgan C G 1969 Lens aberration effects in optical-frequency breakdown of gases Phys. Rev. Lett. 22 1099-102

[19] Pendry J B 2000 Negative refraction makes a perfect lens Phys. Rev. Lett. 85 3966-9

[20] Fleury R, Sounas D L and Alù A 2014 Negative refraction and planar focusing based on parity-time symmetric metasurfaces Phys. Rev. Lett. 113023903

[21] Monticone F, Valagiannopoulos C A and Alù A 2015 'Aberration-Free Imaging Based on Parity-Time Symmetric Nonlocal Metasurfaces,' under review, online on (http:// arxiv.org)

[22] Bender C M 2007 Making sense of non-Hermitian Hamiltonians Rep. Prog. Phys. 70 947-1018

[23] Makris K G, El-Ganainy R, Christodoulides D N and Musslimani Z H 2008 Beam dynamics in PT symmetric optical lattices Phys. Rev. Lett. 100103904

[24] Rüter C E, Makris K G, El-Ganainy R, Christodoulides D N, Segev M and Kip D 2010 Observation of parity-time symmetry in optics Nat. Phys. 6 192-5

[25] Longhi S 2010 PT-symmetric laser absorber Phys. Rev. A 82 031801

[26] Chong Y D, Ge L and Stone A D 2011 PT-symmetry breaking and laser-absorber modes in optical scattering systems Phys. Rev. Lett. 106093902

[27] Lin Z, Ramezani H, Eichelkraut T, Kottos T, Cao H and Christodoulides D N 2011 Unidirectional invisibility induced by PT-symmetric periodic structures Phys. Rev. Lett. 106213901

[28] Regensburger A, Bersch C, Miri M-A, Onishchukov G, Christodoulides D N and Peschel U 2012 Parity-time synthetic photonic lattices Nature 488 167-71

[29] Hodaei H, Miri M-A, Heinrich M, Christodoulides D N and Khajavikhan M 2014 Parity-time-symmetric microring lasers Science 346 975-8

[30] Feng L, Wong Z J, Ma R-M, Wang Y and Zhang X 2014 Single-mode laser by parity-time symmetry breaking Science 346 972-5

[31] Fleury R, Sounas D L and Alù A 2015 An invisible acoustic sensor based on parity-time symmetry Nat. Commun. 6 5905

[32] Valagiannopoulos C A 2011 Electromagnetic scattering of the field of a metamaterial slab antenna by an arbitrarily positioned cluster of metallic cylinders Prog. Electromagn. Res. 114 51-66

[33] Abramowitz M and Stegun I 1972 Handbook of Mathematical Functions with Formulas, Graphs, and Mathematical Tables (New York: Dover) p 360

[34] Chong Y D, Ge L, Cao H and Stone A D 2010 Coherent perfect absorbers: time-reversed lasers Phys. Rev. Lett. 105 053901

[35] Sounas D L, Fleury R and Alù A 2015 Unidirectional cloaking based on metasurfaces with balanced loss and gain Phys. Rev. Appl. 4014005

[36] Valagiannopoulos C A, Tukiainen A, Aho T, Niemi T, Guina M, Tretyakov S A and Simovski C R 2015 Perfect 
magnetic mirror and simple perfect absorber in the visible spectrum Phys. Rev. B 91115305

[37] Pozar D M 1998 Microwave Engineering (New York: Wiley) p 3

[38] Silva A, Monticone F, Castaldi G, Galdi V, Alù A and Engheta N 2014 Performing mathematical operations with metamaterials Science 343 160-3
[39] Monticone F, Estakhri N M and Alù A 2013 Full control of nanoscale optical transmission with a composite metascreen Phys. Rev. Lett. 110203903

[40] Estakhri N M and Alù A 2014 Manipulating optical reflections using engineered nanoscale metasurfaces Phys. Rev.B 89

[41] Tretyakov S A 2015 Metasurfaces for general transformations of electromagnetic fields Phil. Trans. R. Soc. A 373 